\title{
TYPES OF DIABETES
}

\author{
*P.Rajalakshmy
}

\section{Abstract:}

The number of people with diabetes and pre-diabetes are exponentially increasing worldwide due to the population growth, aging, urbanization, unhealthy eating habits, increasing prevalence of obesity and physical inactivity.There are numerous type of diabetes which is not known by many health professions. It is very important to gain knowledge on recently diagnosed diabetes, this diabetes affects both children and adults. Thus this study explains about the some of the recent identified diabetes.

Key words: 1.5 Diabetes mellitus, type 3 diabetes mellitus,Maturity onset diabetes of the young(MODY)

\section{INTRODUCTION}

Worldwide, Diabetes mellitus is a leading cause of morbidity and mortality, it is also associated with life threatening and potentially disabling macro- and microvascular complications ${ }^{1}$. Diabetes is a disease that occurs when your blood glucose, is too high. Blood glucose is your main source of energy and comes from the food you eat. Insulin, a hormone made by the pancreas, helps glucose from food get into your cells to be used for energy. Sometimes your body doesn't make enough insulin or doesn't use insulin well. Glucose then stays in your blood and doesn't reach your cells. Over time, having too much glucose in your blood can cause health problems. Although diabetes has no cure, you can take steps to manage your diabetes and stay healthy. Sometimes people call diabetes "a touch of sugar" or "borderline diabetes." These terms suggest that someone doesn't really have diabetes or has a less serious case, but every case of diabetes is serious.This study will deals with less common Types include Type 1.5, type 3, Maturity onset diabetes of the young(MODY), Brittle diabetes. ${ }^{2}$

\section{Type 1 Diabetes}

Type 1 diabetes, which used to be called juvenile diabetes, your body does not produce insulin or produces very little. Type 1 diabetes usually develops in children and young adults and accounts for 5 to 10 percent of diabetes cases in the United States. Symptoms may include thirst, frequent urination, increased hunger, unexplained weight loss, blurry vision, and fatigue. People who have type 1 diabetes need to take insulin injections daily to make up for what their pancreas can't produce.

\section{Type 2 Diabetes: Insulin Resistance}

Type 2 diabetes, which used to be called adult-onset diabetes, is the most common form of diabetes, accounting for 90 to 95 percent of 
diabetes cases. While most people who develop type 2 diabetes are older, the exact cause of type 2 diabetes is largely unknown, but the disease tends to develop in people who are obese and physically inactive. People who have a family history of diabetes or a personal history of gestational diabetes are also at increased risk of developing type 2 diabetes. Treatment for Type 2 diabetes usually includes dietary changes, regular physical activity, and oral diabetes medications to help control blood glucose. If left untreated, serious health conditions such as heart disease or stroke can develop.

\section{Gestational Diabetes}

Gestational diabetes is a condition that occurs in 3 to 8 percent of pregnant women during late pregnancy. Its cause is thought to be pregnancy-related hormonal fluctuations and a shortage of insulin that often occurs during pregnancy. Many women with gestational diabetes have no symptoms, so it is important to get screened for this condition during pregnancy. Gestational diabetes can lead to problems such as high-birth-weight babies, breathing problems in the baby, and high blood pressure in the mother during pregnancy. Women who have had gestational diabetes have a 40 to 60 percent chance of developing Type 2 diabetes within 5 to 10 years after their pregnancy.

\section{TYPE 1.5 DIABETES}

Type 1.5 Diabetes (T1.5D) is also known as Latent Autoimmune Diabetes of Adults (LADA). LADA is considered by some experts to be a slowly progressive form of Type 1 Diabetes (T1D) while other experts in the field consider it a separate form of Diabetes.LADA or T1.5D is sometimes thought of as T1D that is diagnosed in adults over the age of $30-\mathrm{T} 1 \mathrm{D}$ is commonly diagnosed in children and younger adults. T1.5D is often found along with Type 2 Diabetes (T2D) up to $25 \%$ of individuals with T1.5D also have characteristics of T2D. ${ }^{1}$ this is sometimes called "Double Diabetes". ${ }^{3}$

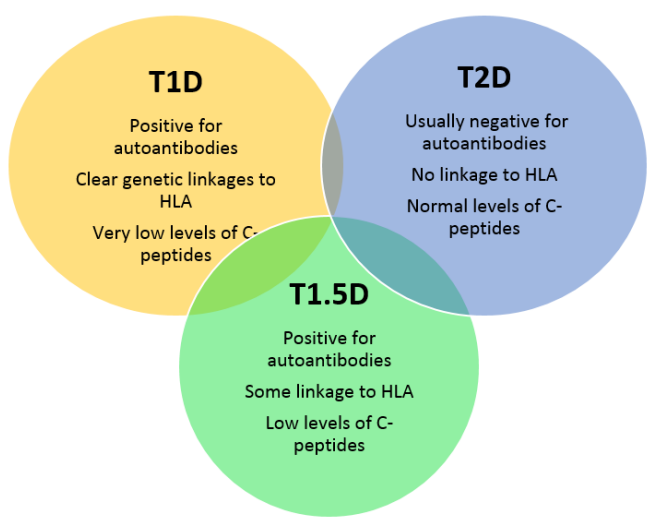

\section{BRITTLE DIABETES}

Brittle diabetes has a number of potential causes. It can be caused by absorption problems in the intestines. This includes delayed stomach emptying, drug interactions, insulin absorption issues and malfunctioning hormones. Severely low blood sugar levels may also create thyroid and adrenal gland problems.

Gastroperesis, delayed stomach emptying, can affect the rate at which food, glucose and insulin is absorbed into the bloodstream. Brittle diabetes is often associated with psychological issues such as stress and depression. With brittle diabetes, however, the fluctuations are more serious and tend to result in frequent hospital visits, interruption to employment and can often contribute to psychological issues such as stress. The life expectancy for someone with brittle diabetes is no different to someone who has type 1 or type 2 diabetes. Brittle diabetes is rare but serious. Around 3 in 1,000 people with type 1 diabetes mellitus will develop brittle diabetes.4

\section{MODY}

"Maturity onset diabetes of the young" (MODY) refers to any of several hereditary 
forms of diabetes mellitus caused by mutations in an autosomal dominant gene[2] disrupting insulin production. MODY is often referred to as "Monogenic Diabetes" to distinguish it from the more common types of diabetes (especially type 1 and type 2), which involve more complex combinations of causes involving multiple genes and environmental factors. MODY 2 and MODY 3 are the most common forms. 5 MODY should not be confused with latent autoimmune diabetes of adults (LADA) . ${ }^{5}$

\section{TYPE 3 DIABETES MELLITUS:}

Type 3 diabetes mellitus (T3DM) corresponds to a chronic insulin resistance plus insulin deficiency state that is largely confined to the brain, can overlap with T2DM.It has proposed that T3DM represents a major pathogenic mechanism of Alzheimer's disease (AD) neurodegeneration. ${ }^{6}$

Interest in clarifying the roles of T2DM, insulin resistance, and hyperinsulinemia in relation to cognitive impairment primary brain insulin resistance and insulin deficiency mediate cognitive impairment and AD. ${ }^{7}$

\section{CONCLUSION:}

Diabetes is a disorder which need to be controlled there are many types of newly diagnosed diabetes which is not yet found with a proper treatment, still more research need to be done with this different types of diabetes.

\section{REFERENCES}

1. Levin M, Pfeifer M: Uncomplicated Guide to Diabetes Complications. 3rd edition. Alexandria, Virginia: American Diabetes Association 2009.
2. Stenström G., Gottsäter A., Bakhtadze E., Berger B., Sundkvist G. (December 2005). "Latent autoimmune diabetes in adults: definition, prevalence, beta-cell function, and treatment". Diabetes. 54 Suppl 2: S68-72.

3. American Diabetes, Association (January 2007). "Diagnosis and classification of diabetes mellitus". Diabetes Care. 30 Suppl 1: S42-7.

4. Edghill, EL; Bingham, C; Slingerland, AS; Minton, JA; Noordam, C; Ellard, S; Hattersley, AT (December 2006). "Hepatocyte nuclear factor-1 beta mutations cause neonatal diabetes and intrauterine growth retardation: support for a critical role of HNF-1beta in human pancreatic development". Diabetic Medicine. 23 (12): 1301-6.

5. Jellinger KA. Neuropathological aspects of Alzheimer disease, Parkinson disease and frontotemporal dementia. Neurodegener Dis. 2008;5(3-4):118-121.

6. Rivera EJ, Goldin A, Fulmer N, Tavares R, Wands JR, de la Monte SM. Insulin and insulin-like growth factor expression and function deteriorate with progression of Alzheimer's disease: link to brain reductions in acetylcholine. J Alzheimers Dis. 2005;8(3):247-268.

7. Yorifuji, T; Kurokawa, K; Mamada, M; Imai, T; Kawai, M; Nishi, Y; Shishido, S; Hasegawa, Y; Nakahata, T (June 2004). "Neonatal diabetes mellitus and neonatal polycystic, dysplastic kidneys: Phenotypically discordant recurrence of a mutation in the hepatocyte nuclear factor-1beta gene due to germlinemosaicism". The Journal of Clinical Endocrinology and Metabolism. 89 (6): 2905-8. 